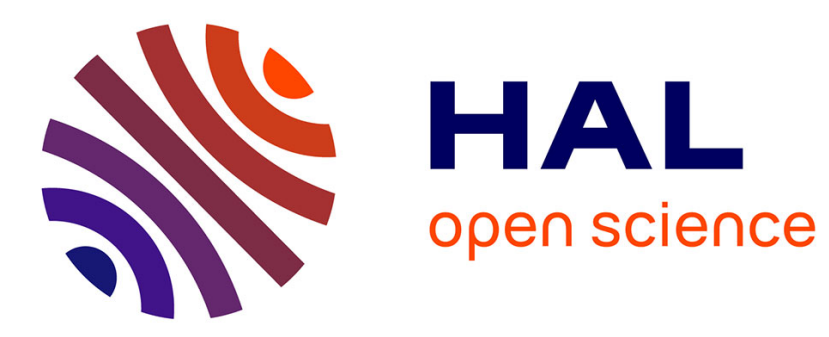

\title{
Energy Efficient Multi-Flow Routing in Mobile Sensor Networks.
}

Nicolas Gouvy, Essia Hamouda Elhafsi, Dimitrios Zorbas, Nathalie Mitton

\section{To cite this version:}

Nicolas Gouvy, Essia Hamouda Elhafsi, Dimitrios Zorbas, Nathalie Mitton. Energy Efficient MultiFlow Routing in Mobile Sensor Networks.. IEEE WCNC - Wireless Communications and Networking Conference, Apr 2013, Shanghai, China. hal-00772217

\section{HAL Id: hal-00772217 https://hal.inria.fr/hal-00772217}

Submitted on 4 Jun 2013

HAL is a multi-disciplinary open access archive for the deposit and dissemination of scientific research documents, whether they are published or not. The documents may come from teaching and research institutions in France or abroad, or from public or private research centers.
L'archive ouverte pluridisciplinaire HAL, est destinée au dépôt et à la diffusion de documents scientifiques de niveau recherche, publiés ou non, émanant des établissements d'enseignement et de recherche français ou étrangers, des laboratoires publics ou privés. 


\title{
Energy Efficient Multi-Flow Routing in Mobile Sensor Networks
}

\author{
Nicolas Gouvy ${ }^{1,3}$, Essia Hamouda ${ }^{2,3}$, Nathalie Mitton ${ }^{3}$ and Dimitrios Zorbas ${ }^{3}$ \\ ${ }^{1}$ Université Lille 1, LIFL (nicolas.gouvy@lifl.fr) ${ }^{2}$ California State University, Fullerton, ${ }^{3}$ Inria Lille-Nord Europe
}

\begin{abstract}
Controlled mobility is one of the most complex challenges in Wireless Sensor Networks (WSN). Only a few routing protocols consider controlled mobility in order to extend the network lifetime. They are all designed to optimize the physical route topology from a source to a destination. However, there is often more than one sensor which reports an event to the sink in WSN. In existing solutions, this leads to oscillation of nodes which belong to different routes and their premature death. Experiments show that the need of a routing path merge solution is high. As a response we propose the first routing protocol which locates and uses paths crossing to adapt the topology to the network traffic in a fully localized way while still optimizing energy efficiency. Furthermore the protocol makes the intersection to move away from the destination, getting closer to the sources, allowing higher data aggregation and energy saving. Our approach outperforms existing solutions and extends network lifetime up to $37 \%$.
\end{abstract}

\section{INTRODUCTION}

Miniaturization, cost decrease and advances in low-power electronics and radio communication technologies have made possible the emergence of Wireless Sensor Networks (WSN). WSN are cooperative sets of battery-powered nodes which communicate through radio links and can be employed for a wide kind of applications, gathering data from their environment on-request or on event occurrence. WSN are intended to be used in vast applications in hostile environments such as battlefield or surveillance systems. The importance of the applications makes essential to propose routing algorithms which take the battery issue into account in order to improve the network lifetime. A common solution to tackle the fast energy exhaustion is to increase sensor density in order to decrease communication range. A more recent approach is to introduce mobility-enabled sensors, that can move to areas where resources are most needed in order to efficiently route packets. Results in [1] show that deploying resourcefull mobile devices in a WSN provides better results than increasing the network density.

Still, only a few works consider the use of controlled mobility in order to optimize route topology. Moreover, even if they differ in the relocation patterns they use, they all aim to relocate nodes on the source to the destination route. Nevertheless, in event-based WSN, there are often multiple sources emitting at the same time to the sink. It turns out that existing routing protocols which relocate nodes do not work well in this case. They provoke useless zig-zag movements when routes are close and consequently create holes in the network topology when multiple routes cross each other [2].

In this paper we propose PAMAL (PAth Merging ALgorithm), a new geographic routing algorithm for WSN with mobile nodes, that takes advantages of paths crossing and even favors it in the following respect:

- It guarantees network connectivity at each step.

- It is energy efficient as it takes both radio and relocation costs into account when relocating nodes.

- It is localized as routing decisions are based only based on neighbors and destination locations.

- It detects path crossing and achieves better network lifetime than existing solutions.

Simulations show that PAMAL stops oscillation and makes close routing paths merge ahead to the destination from time to time, since it moves the intersection point close to the sources. This behavior and data-aggregation makes PAMAL improve network lifetime up to $37 \%$ compared to the best of our knowledge routing protocol which uses controlled mobility [3].

The remainder of this paper is organized as follows. In Section 2, we provide a review of existing works on routing algorithms which use controlled mobility and data-aggregation. We detail the models needed for the rest of the paper in Section 3. The preliminaries are exposed in Section 4, while Section 5 describes our motivation. PAMAL routing algorithm and how path crossing is managed is introduced in Section 6. In Section 7, we describe our simulation methodology and present our simulation results. Finally Section 8 concludes the paper.

\section{RELATED WORK}

\section{A. Position-based routing in static networks.}

Position based routing algorithms for static sensor networks have been widely studied in the literature. In distance-based greedy routing a node currently holding a packet forwards it to the closest neighbor to the destination. This method has been extended to support energy efficiency [4], guaranteed packet delivery [5] or both [6]. The Cost Over Progress (COP) based routing introduced in [4] is a localized metric-aware greedy

This work was partially supported by CPER Nord-Pas-de-Calais/FEDER Campus Intelligence Ambiante and the ANR BinThatThinks project. 
routing scheme. A node forwards a packet to its neighbor in the direction of the destination such that the ratio of the energy consumed for forwarding packet (any cost metric can be used) to the progress made (measured as the reduction in distance up to the destination) is minimized.

\section{B. Routing in mobile networks}

Node mobility is generally regarded as a hazard for geographic routing, causing a degradation of performances or even persistent routing failures. Therefore, little work has been done in controlled mobility assisted routing in WSN. It is generally used to distribute traffic load and consequently optimize energy consumption [7]. However those protocols do not alter the network topology. Other approaches adopt existing routing protocols to find an initial route, and iteratively move each node to the midpoint of its upstream and downstream nodes on the route. These routing protocols may not be efficient. For example moving strategy in [7] may cause useless zigzag movements. In MobileCOP [2], next hop on the path is selected based on COP metric [4]. Once a path is computed, its nodes are moved and placed equidistantly on the straight line connecting the source to the destination. Such a strategy may induce a memory overhead on nodes and a high transmission delay, since they have to store the full path. Moreover, the network may be disconnected (a node may move out of range of its neighbor). CoMNet [3] or [8] is the first fully localized COP-based geographic routing protocol that takes the cost of moving into account while it guarantee network connectivity. Nevertheless, to the best of our knowledge, none of existing solutions handles paths crossing. In all those protocols, an Intersection Node $(I N)$ between two different crossing paths will be relocated continuously on each source-destination line at each routing. We propose PAMAL which takes intersection into account, and handles it dynamically and provokes merging of different close routing paths to the same destination in order to make energy savings and data-aggragation.

\section{In-Network data aggregation}

Data-aggregation algorithms combine gathered data coming from different sources to eliminate redundancy or lower the routing overhead in order to minimize the size and/or number of radio transmissions and consequently make possible energy saving. For a complete survey, readers should refer to [9].

\section{SYSTEM DESCRIPTION}

\section{A. Assumptions}

We consider a sensor network where nodes are randomly and uniformly scattered. It can be modeled as an undirected simple finite graph $G(V, E)$, with $V$ the set of nodes in the network and $E$ the set of links/edges. $(u, v) \in E$ if $u$ and $v$ are in transmission range of each other. We denote by $N(u)$ the set of neighbors of $u$. Let $\delta(u)=|N(u)|$ be the cardinality of $N(u)$, also called the degree of node $u$. We also define $N_{D}(u)$ the subset of neighbors of node $u$ which are closer to the destination node $D$ than $u: N_{D}(u)=$ $\{v \in N(u) \wedge|v D|<|u D|\}$.

We assume that every node $v \in V$ is aware of its geographical location. Moreover each node can be either a mobile or stationary sensor. The latter assumption can be further relaxed by making either all nodes static or mobile.

\section{B. Energy models}

Although our approach is model independent, we use the following widely employed radio and mobility cost models as a proof of concept.

1) Transmission cost: We denote by $C_{s}($.$) the energy$ consumption or cost for radio transmission between two nodes [10]:

$$
C_{s}(r)=q *\left(r^{\alpha}+c\right) \quad \text { if } r \neq 0,
$$

where $c$ represents the energy overhead due to radio device, $\alpha$ is a real constant $(>1)$ that represents the signal attenuation, and $q$ represents the size of the packet transmitted in bits.

According to this model, the optimal radio transmission radius for routing is $\mathrm{r}^{*}=\sqrt[\alpha]{\frac{\mathrm{c}}{\alpha-1}}[11]$.

2) Mobility cost: We denote by $C_{m}($.$) the cost to relocate a$ node. We use the model adopted in previous similar works [2]:

$$
C_{m}\left(\left|v v^{\prime}\right|\right)=a *\left|v v^{\prime}\right|
$$

where $v$ is the initial position of $v$ (before it is moved) and $v^{\prime}$ is its new position. $a$ is a constant defining the units of energy consumed per units of distance traveled.

\section{PRELIMINARIES}

PAMAL relies on the CoMNet (COnnectivity preservation Mobile routing protocols for actuator and sensor NETworks) [3] routing protocol.

CoMNet uses a COP approach: current node $u$ chooses $v \in N_{D}(u)$ which minimizes the ratio of the global cost (packet transmission cost plus node relocation cost) to the progress made towards the destination. Indeed, $v$ satisfies the following optimization problem:

$$
v=\arg _{\min _{v \in N_{D}(u)}} \frac{\mathrm{C}_{\mathrm{s}}(|u v|)+\mathrm{C}_{\mathrm{m}}\left(\left|\mathrm{vv}^{\prime}\right|\right)}{|u D|-\left|v^{\prime} D\right|}
$$

The connectivity of the network is guaranteed using a Connected Dominating Set (CDS) and $v=v^{\prime}$ if $v$ belongs to the CDS.

In order to adapt to various cost models CoMNet comes with three different variants -more details can be found in [3]- :

- CoMNet-ORouting on the Move which aligns nodes on a straight line from $S$ to $D$ (SD),

- CoMNet - Move $(D S r)$ which aligns nodes on (SD) line with all hop lengths to be equal to the optimal transmission distance $r^{*}$,

- CoMNet-Move $_{r}$ in which next hop node $v$ is relocated on the intersection of the circle $C_{r^{*}}$ of radius $r^{*}$ centered at $u$ and the (vD) line. 


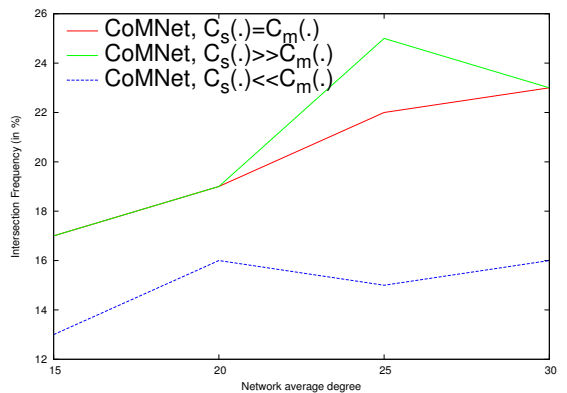

Fig. 1: Path crossing frequency (in \%) with regards to network degree and cost model.

\section{Motivation}

The motivation of this work is to take advantage of node mobility and path crossing to extend the network lifetime. Currently even though the idea of using mobile sensors to optimize network topology begins to be recognized, all current approaches still rely on the strong hypothesis of a unique source-destination pair.

Available solutions iteratively move each node of the SourceDestination $(S, D)$ route on a $(S D)$ line. However in eventbased WSN, a same event oftenly triggers multiple sensors in a close area, provoking competition and useless zig zag for nodes which belong to the different $\left(S_{1}, D\right) \ldots\left(S_{n}, D\right)$ routes.

In order to demonstrate the need for path crossing management, we perform simulation on random topologies of various densities $\delta(\delta=15,20,25,30)$ with only two source $S_{1}$ and $S_{2}$ and one sink $D$. Routing is performed on those networks between each pair of source-destination $\left(S_{1}, D\right)$ and $\left(S_{2}, D\right)$ using CoMNet. Figure 1 shows that intersections frequency vary from $13 \%$ and up to $20 \%$ of network topologies depending on network density. Those results vindicate the necessity of dynamic path crossing management as we can infer a higher intersection rate in real environment where sources are close.

\section{PAMAL}

We propose PAMAL which takes advantage of paths crossing. We argue that making the different routes merge -in a fully localized way- provide energy saving since less nodes are sollicited. When a node detects that it is an intersection node (IN), i.e. a node which receives packets from two different sources for a same direction, it broadcasts the information to its 1-hop neighborhood. Each of its neighbors $v$ will stop considering the sink $D$ as final destination for its relocation pattern computation but will use the $(v, I N)$ route instead. From time to time, route merging can be done closer to the sources up to a stable state in a purely localized way. Note that for the sake of clarity we focus on the case of two different sources with a unique sink, however PAMAL could easily be extended to $n$-sources.

Figure 2 illustrates the case in which two source nodes, $S_{1}$ and $S_{2}$ route packets toward $D$. Depending on network settings and locations of $S_{1}$ and $S_{2}$, a specific node will receive packets

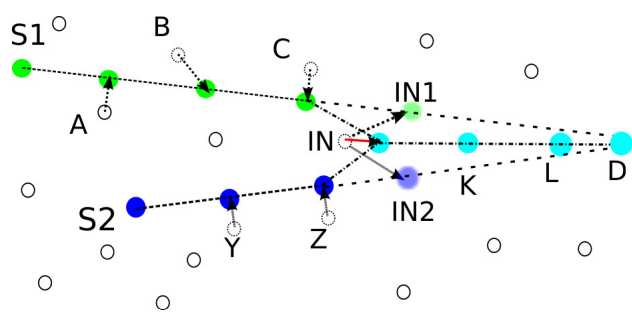

Fig. 2: Source nodes $S_{1}$ and $S_{2}$ route data up to sink $D$. Routing paths intersect on $I N$ node.

from both sources. It becomes an $I N$ that will aggregate data from both sources. Starting from $I N$, the $(I N, D)$ route belongs to both $\left(S_{1}, D\right)$ and $\left(S_{2}, D\right)$.

We can observe that source node $S 1$ routes packet towards $D$ by relocating successively nodes $A, B$ and $C$ on the $\left(S_{1} D\right)$ line. At the same time source node $S_{2}$ routes packet towards $D$ by relocating successively nodes $Y$ and $Z$ on the $\left(S_{2} D\right)$ line. However, nodes $C$ and $Z$ belong to different routing paths provoke oscillation. Node $C$ aims to relocate the $I N$ node on the $I N 1$ location while node $Z$ asks $I N$ to relocate itself on the $I N 2$ location. Both $C$ and $Z$ are in competition for the same node, $I N$. In existing solutions $I N$ would be relocated successively on $\left(S_{1}, D\right)$ in $I N 1$ or $\left(S_{2}, D\right)$ in $I N 2$ for each received packet and would provoke oscillation. In PAMAL, the IN will:

- be re-localized on the iso-barycenter of its original, IN1 and $I N 2$ locations and refuse to move while it is an IN,

- advertize its one-hop neighbors that they should target it as the destination for their relocation pattern.

- aggregate packet from both sources and route them towards the destination.

Consequently, $(S 1, D)$ and $(S 2, D)$ tend to be reformed as $(S 1, I N, D)$ and $(S 2, I N, D)$ respectively. On path $(I N, K$, $L, D)$ aggregated packets are routed. Depending on the distance between the sources and between each source and the destination, the IN moves toward the sources, extending the length of the shared path $(I N, D)$. In the next subsection we detail how an IN is elected.

\section{A. Intersection Node election process}

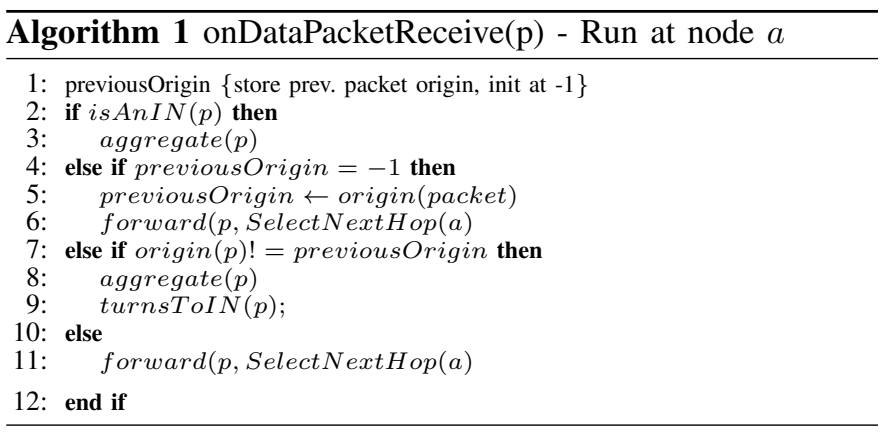

PAMAL relies on the use of $I N$ which performs packet aggregation and serves as a temporary destination for its 


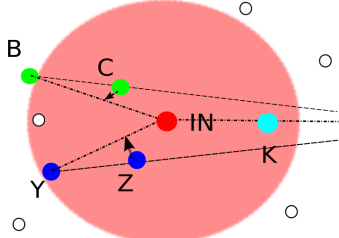

Fig. 3: The $I N$ broadcasts its existence to its 1-hop neighborhood. As a consequence they do not consider $D$ location for their relocating pattern anymore

neighbors in their relocation patterns computation.

An $I N$ appears dynamically when a node has noticed that it has recieved packets to route from different sources (Alg. 1, Line 7). It advertises its neighbors (Alg. 1, Line 9) to use it as final destination in their relocation pattern computation (Figure 3) instead of $D . I N$ starts aggregating the packets (Alg. 1, Line 8) and forwards them. From time to time, the paths will tend to merge and a new $I N$ may appear ahead to the destination (node $C$ or $Z$ Figure 3). An $I N$ node turns back to standard node as soon as it receives already aggregated packets. Its neighbors stop to target it as destination in their relocation pattern computation. As we propose a general purpose routing protocol, the data aggregation mechanism we use is minimalistic. A more advanced data aggregation could be performed with respect to the data and/or application. Aggregated and non aggregated packets are routed using Algorithm 2. Routing process is detailled in Section VI-B.

\section{B. Routing process}

Unlike previous solutions, the next hop selection does not simply use one relocation pattern for the next hop computation. On the contrary, current node $a$ holding a packet computes the COP of the relocation of every node $v$ in $N_{D}(a)$ with each relocation pattern presented in Section IV. This requires higher computing complexity but reduces energy cost. A node $a$ holding a packet execute Alg. 2. First, $a$ checks whether it has been notified for an IN node for this source/destination pair. If true (Alg. 2, line 4) $a$ will use the IN location (Alg. 2, line 5) instead of $D$ location (see Figure 3 ) in its relocation pattern. Then $a$ computes for each $v$ in $N_{D}(a)$ the COP and relocation according to the three different CoMNet relocation pattern (Alg. 2, lines 11-14). a stores the next hop $v$ which minimizes the COP and its relocation (Alg. 2, lines 15-20) and return it (Alg. 2, line 22). In any case, routing fails if there is no neighboor closer to $D$ (Alg. 2, lines 7-9). PAMAL relies on the CDS introduced by CoMNet to guarantee network connectivity.

\section{ViI. Performance Evaluation}

In this section, we evaluate the performance of PAMAL compared to CoMNet. We do not compare to other routing protocols which use controlled mobility as CoMNet already outperfomed them [3].

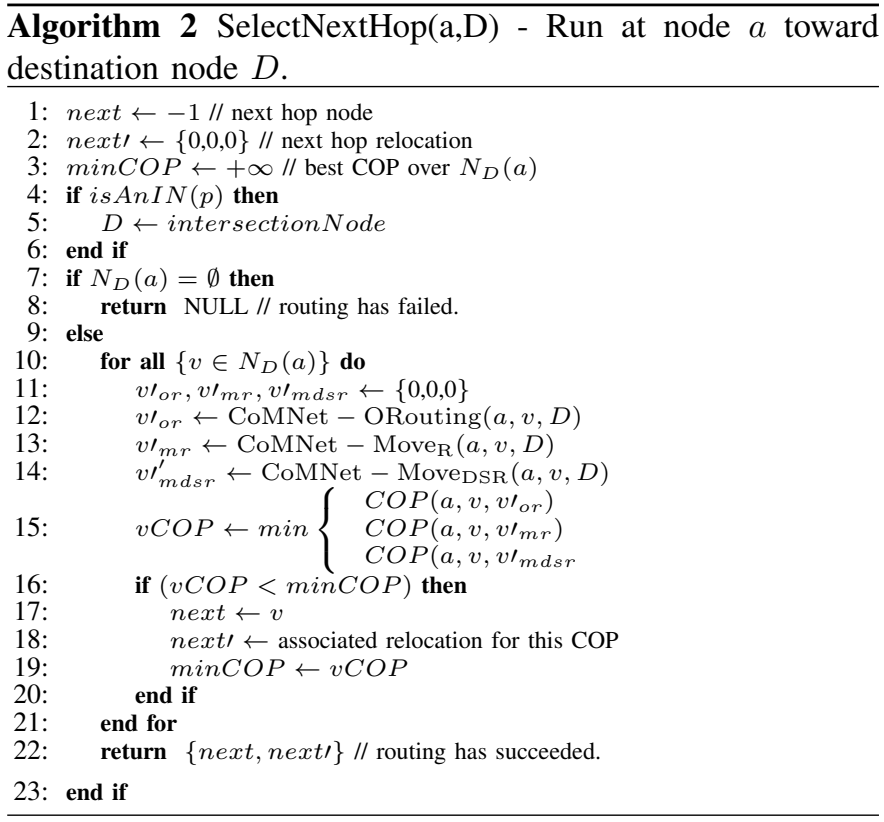

\section{A. Simulation Setup}

We perform simulations using the last release of WSNet [12] network simulator on randomly generated maps where two different sources target one destination and their routing path crosses. Maximal radio range of nodes is set to $50 \mathrm{~m}$. The MAC header size is set to 40Bytes and packet size is either 200Bytes or 400Bytes. Aggregation factor is of 1:2. Node speed is set to $1 \mathrm{~m} . s^{-1}$. To compute $C_{s}($.$) , we use$ common values, i.e. $c=3 \times 10^{-} 9 \mathrm{~J}$. and $\alpha=2$, which lead to an $r^{*}=22.36 \mathrm{~m}$. Regarding the mobility model parameter $a$ (see Eq. 2), we run simulations with the following options :

1) if sending cost equals the moving, $C_{s}()=.C_{m}(),$.$a is$ solution to the equation $C_{s}\left(r^{*}\right)=C_{m}\left(r^{*}\right)$.

2) if sending cost is much higher than moving cost, $C_{s}()>.>C_{m}($.$) , then a$ is solution to $C_{s}\left(r^{*}\right)=$ $10^{2} C_{m}\left(r^{*}\right)$.

3) if moving cost is much higher than sending cost, $C_{s}()<.<C_{m}($.$) , then a$ is solution to $C_{s}\left(r^{*}\right)=$ $10^{-2} C_{m}\left(r^{*}\right)$.

\section{B. Topology changes}

Figures 4 and 5 illustrate the average length of the common part of the two paths in terms of number of hops as a function of time. Results show that PAMAL provides effective path merging and those merged parts are significantly longer than in CoMNet. More precisely, the path merging at the beginning is significantly quicker and higher in PAMAL thanks to the IN advertising regardless the energy cost model or packet size, until death of nodes. When nodes begin to die, path merging turns to be low while it raises again as the IN selection process takes some time in order to adapt to the network topology. With respect to network density, results show that the lower 


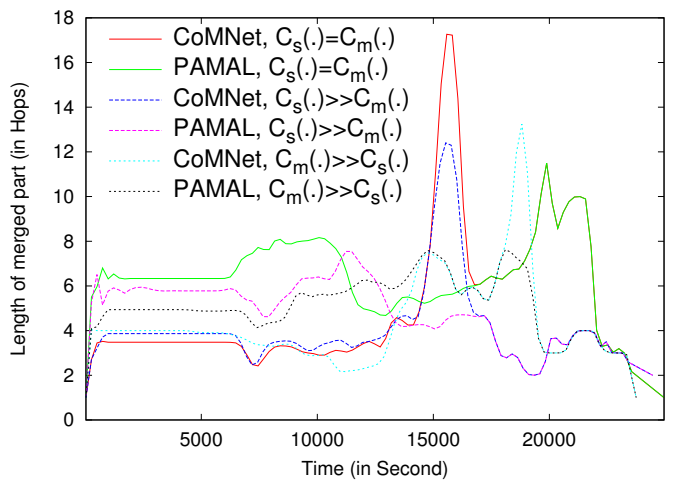

(a) $\delta=15$, Packet size is 200 Bytes.

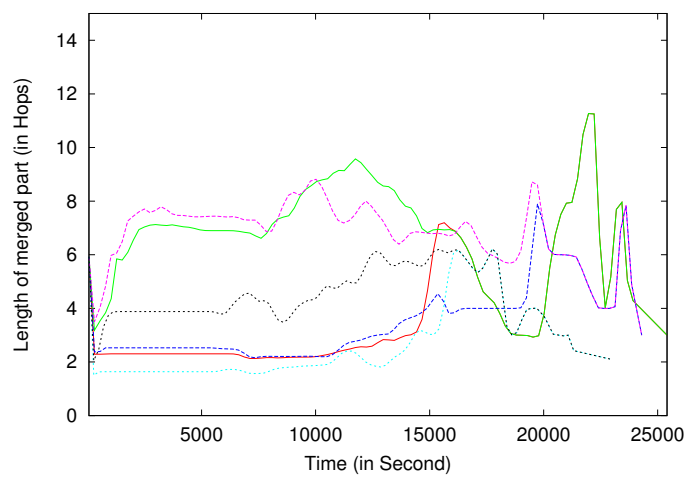

(b) $\delta=20$, Packet size is 200Bytes.

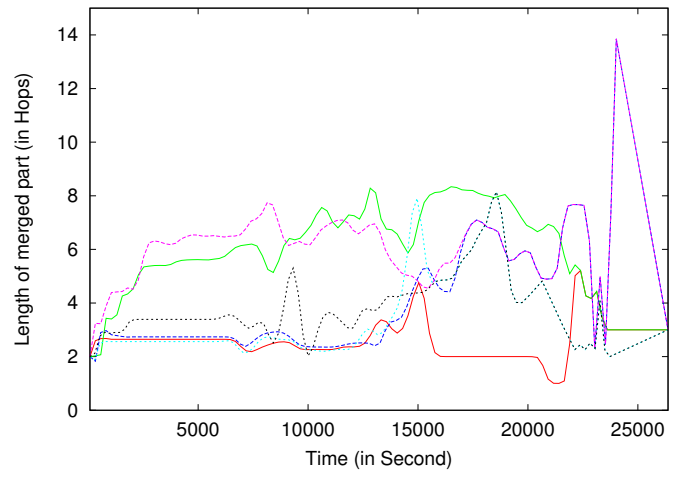

(c) $\delta=25$, Packet size is 200Bytes.

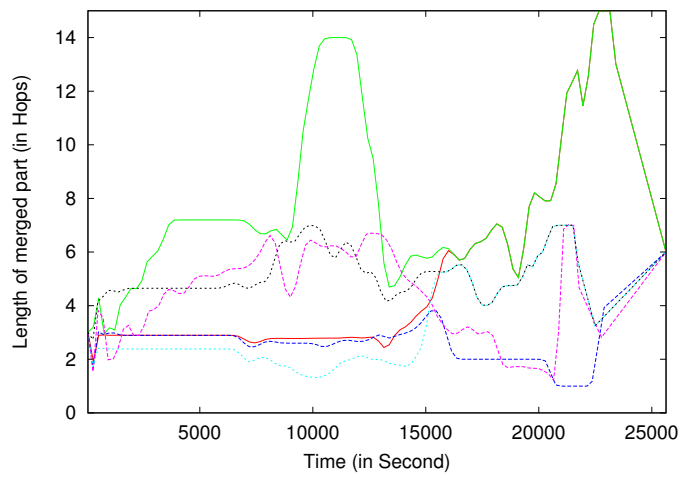

(d) $\delta=30$, Packet size is 200Bytes.

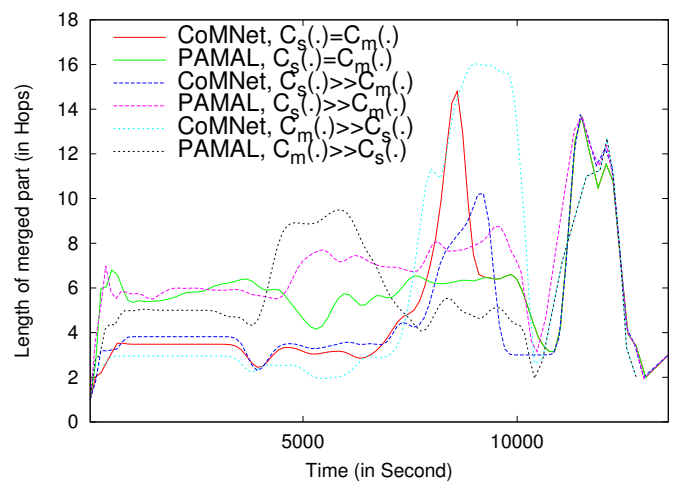

(a) $\delta=15$, Packet size is 400 Bytes.

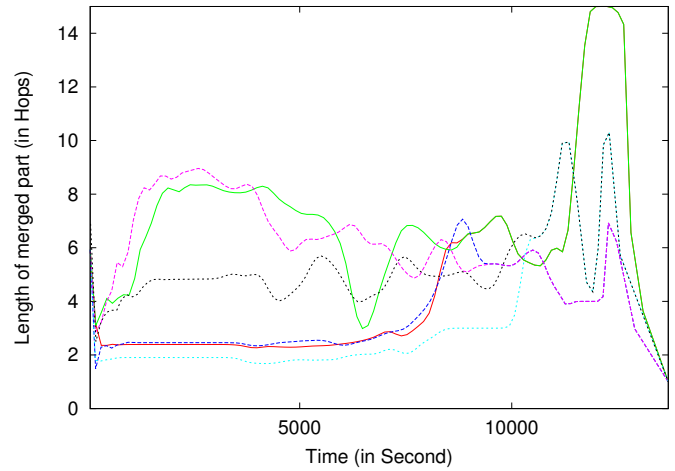

(b) $\delta=20$, Packet size is 400 Bytes.

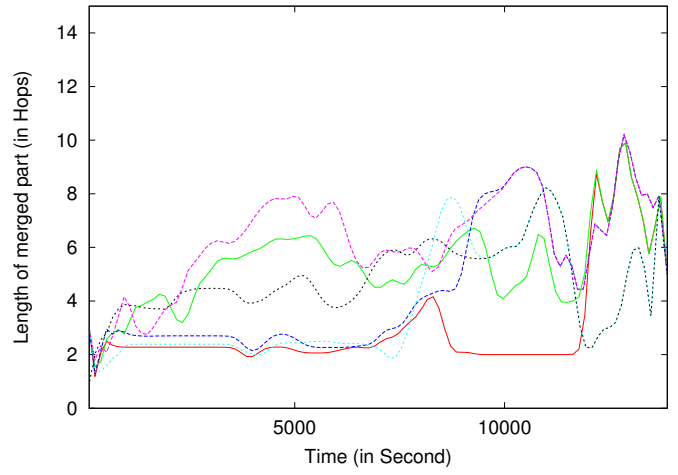

(c) $\delta=25$, Packet size is 400 Bytes.

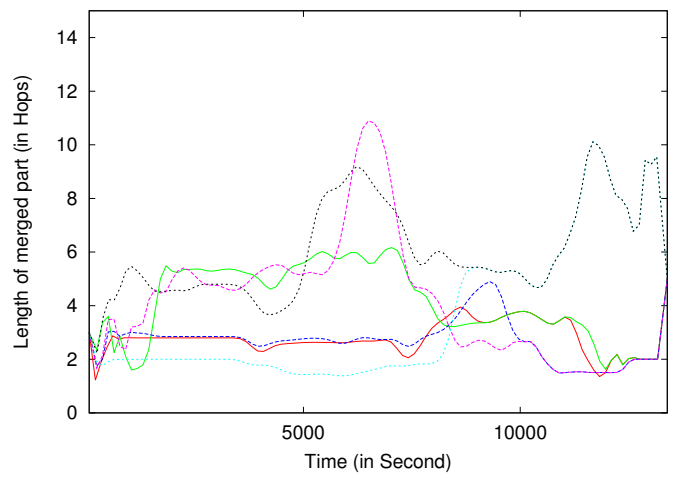

(d) $\delta=30$, Packet size is 400 Bytes.

Fig. 4: Length of merged part of routes with regards to time. Fig. 5: Length of merged part of routes with regards to time. Packet size is 200Bytes. Caption is the same for all figures.

Packet size is 400Bytes. Caption is the same for all figures. 
the network density is, the lower gap between our PAMAL proposal and CoMNet is. This happens because most of the nodes are members of the CDS and thus cannot move. When the density increases $(\delta=20)$, more and more nodes are free to move, and thus the energy gain is much higher. With respect to packet size we observe that merged path length fluctuation is higher with a packet size of 400Bytes than with a packet of 200Bytes. It is reasonable since the larger the packets are the more energy they require. Consequently nodes die quicker and topology changes quickly. With respect to cost model, results show that the improvement is minimal when $C_{s}()<.<$ $C_{m}($.$) compared to the other models as it tends to limit node$ movements.

\section{Energy cost}

Figures 6, 7 and 8 compare the average network lifetime as a function of network density for each of the studied algorithms with an initial packet size of 400Bytes. The network lifetime is defined as the average between reception time of the last packet of route $(S 1, D)$ and $(S 2, D)$. Simulation results show that PAMAL outperforms the other protocols when $C_{s}()=$. $C_{m}($.$) (Figure 6), when C_{s}()>.>C_{m}($.$) (Figure 7) and when$ $C_{s}()<.<C_{m}($.$) (Figure 8). Based on our results we can infer$ that when a sufficient density is reached $(\delta>=20)$ PAMAL achieves a significantly higher network lifetime. The network lifetime extension is maximized when the $C_{s}()<.<C_{m}($. model is used.

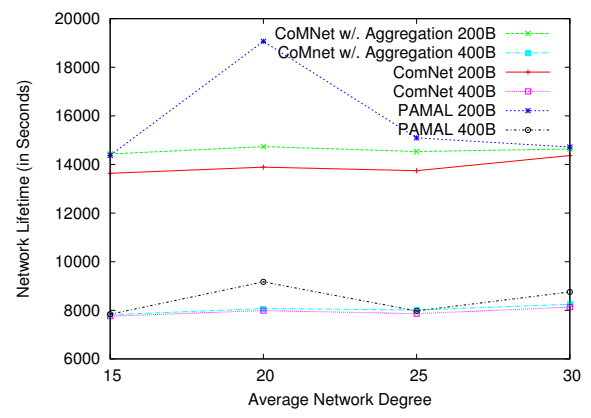

Fig. 6: Length of merged part of routes with regards to time. Packet size is 400 Bytes. $C_{s}()=.C_{m}($.

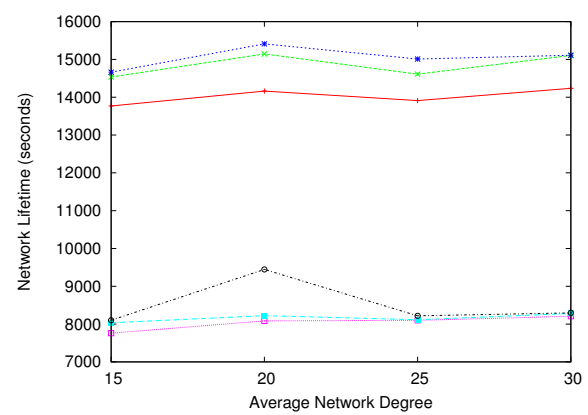

Fig. 7: Length of merged part of routes with regards to time. Packet size is 400 Bytes. $C_{s}()>.>C_{m}($.

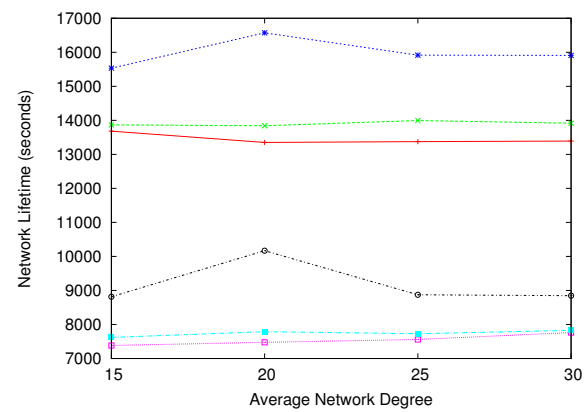

Fig. 8: Length of merged part of routes with regards to time. Packet sie is 400 Bytes. $C_{s}()<.<C_{m}($.

\section{CONCLUSION AND FUTURE WORKS}

In this paper we proposed a new kind of routing protocol which takes mobility into account with path crossing in WSN. The mechanism of route merging we exposed provides significant energy savings regardless of the network density or the packet size. Through extensive simulations we show that our approach increases up to $37 \%$ the network lifetime compared to the best of our knowledge routing protocol relying on controlled mobility. Future work will focus on more complex environment and network topologies and address the hole bypassing problem. REFERENCES

[1] W. Wei, V. Srinivasan, and K.-C. Chua, "Extending the lifetime of wireless sensor networks through mobile relays," IEEE/ACM Trans. on Networking, vol. 16, no. 5, 2008.

[2] H. Liu, A. Nayak, and I. Stojmenovic, "Localized mobility control routing in robotic sensor wireless networks," in Proc. Int. Conference on Mobile Sensor Networks, (MSN), 2007.

[3] E. H. Elhafsi, N. Mitton, and D. Simplot-Ryl, "Energy efficient mobile routing in actuator and sensor networks with connectivity preservation," in $A D H O C-N O W, 2011$.

[4] J. Kuruvila, A. Nayak, and I. Stojmenovic, "Progress and location based localized power aware routing for ad hoc sensor wireless networks," Int. Journal on Distributed Sensor Networks, (IJDSN), vol. 2, 2006.

[5] P. Bose, P. Morin, I. Stojmenovic, and J. Urrutia, "Routing with guaranteed delivery in ad hoc wireless networks," Wireless Networks, vol. 7, 2001.

[6] E. Elhafsi, N. Mitton, and D. Simplot-Ryl, "End-to-end energy efficient geographic path discovery with guaranteed delivery in ad hoc and sensor networks," in Proc. IEEE Int. Symp. on Personal, Indoor and Mobile Radio Communications, (PIMRC), 2008.

[7] D. Goldenberg, J. Lin, and A. Morse, "Towards mobility as a network control primitive," in Proc. ACM Int. Symp. on Mobile Ad Hoc Networking and Computing, (MobiHoc), 2004.

[8] N. Gouvy, E. H. Elhafsi, N. Mitton, and D. Simplot-Ryl, "Minimising energy consumption through mobility with connectivity preservation in sensor networks," The Int. Journal of Parallel, Emergent and Distributed Systems, May 2012.

[9] R. Rajagopalan and P. K. Varshney, "Data-aggregation techniques in sensor networks: a survey," Commun. Surveys Tuts., vol. 8, no. 4, 2006.

[10] V. Rodoplu and T. Meng, "Minimizing energy mobile wireless networks." IEEE Journal in Selected Areas in Communications, (JSAC), vol. 17, no. 8, 1999.

[11] I. Stojmenovic and X. Lin, "Power-aware localized routing in wireless networks," IEEE Trans. on Parallel and Distributed Systems, vol. 12, no. $11,2001$.

[12] A. Fraboulet, G. Chelius, and E. Fleury, "Worldsens: Development and prototyping tools for application specific wireless sensors networks," in Proc. Int. Symp. on Information Processing in Sensor Networks, 2007. 